\title{
Commentary: Piéron's law is not just an artifact of the response mechanism
}

\author{
José M. Medina * and José A. Díaz \\ Departamento de Óptica, Facultad de Ciencias, Edificio Mecenas, Universidad de Granada, Granada, Spain
}

Keywords: human reaction time, power laws, decision making, information entropy, statistical physics, fractals

\section{A commentary on}

Piéron's law is not just an artifact of the response mechanism by Donkin, C., and van Maanen, L. (2014). J. Math. Psychol. 62-63, 22-32. doi: 10.1016/j.jmp.2014.09.006

It has long been known that the mean human reaction/response time $(\mathrm{RT}), t_{R T}$, decreases as the stimulus strength or intensity $S$ increases (Cattell, 1886), reaching an asymptotic value or plateau, $t_{R T_{0}}$, at very high $S$-values in all sensory modalities. A well-established power law, namely, Piéron's law, describes mathematically that empirical relationship (Piéron, 1914; Luce, 1986):

$$
t_{R T}=t_{R T_{0}}+k S^{-p}
$$

Edited by:

Paolo Allegrini,

Consiglio Nazionale delle Ricerche,

Italy

Reviewed by:

Willy Wong,

University of Toronto, Canada

Gerardo Aquino,

Imperial College London, UK

*Correspondence: José M. Medina, jmedinaru@cofis.es

Specialty section: This article was submitted to Fractal Physiology, a section of the journal Frontiers in Physiology

Received: 15 April 2015 Accepted: 16 June 2015 Published: 30 June 2015

Citation:

Medina JM and Díaz JA (2015) Commentary: Piéron's law is not just an artifact of the response mechanism. Front. Physiol. 6:190. doi: 10.3389/fphys.2015.00190 where $k$ and $p$ are coefficients; the latter being a fractional exponent that controls the $R T$ decay. Donkin and van Maanen has investigated the origin of Piéron's law based on a version of the Linear Ballistic Accumulator model. They concluded that Piéron's law is not due only to a decision making process. Various types of models have been proposed for describing the foundations of Piéron's law (Link, 1992; Baird, 1997; Stafford and Gurney, 2004; Hsu, 2005; Palmer et al., 2005; Stafford et al., 2011; Servant et al., 2014; Verdonck and Tuerlinckx, 2014). The model proposed by Donkin and van Maanen belongs to an influential class of models in mathematical psychology, i.e., sequential sampling models. In general, these models of Piéron's law assume the existence of an internal variable threshold. From the stimulus onset, there is an accumulation of noisy "sensory information" or "evidence" until a response criterion is reached. However, the concept of information is not properly defined within the context of information theory and plays no role. A decisional stage is usually implemented in the form of random walk, diffusion, and accumulator models. Despite these models mimic the functional form of Piéron's law, it is not clear whether they are able to explain the internal structure of $k$ and $t_{R T_{0}}$ in Equation (1) and to provide more detailed predictions based on threshold mechanisms. For instance, all these models often postulate that the asymptotic term $t_{R T_{0}}$ is nearly invariant and includes non-decision components (e.g., the motor execution time) that do not hold a chronological order. However, $k$ and $t_{R T_{0}}$ span a range of experimental values and depend on early sensory processing (Pins and Bonnet, 1997; Plainis and Murray, 2000; Murray and Plainis, 2003).

There is an information-theoretic approach, which is rarely mentioned in the literature of Piéron's law, that derives Equation (1) from an optimal information process in sensory perception. In this framework, the first stage of RTs always corresponds to an efficient stimulus encoder. Only after this initial stage there is a bona fide accumulation of information over time, $\Delta H>0$ (e.g., measured in bits), that is related with power law behavior at the threshold, $\beta S_{0}^{p} . S_{0}$, and $\beta$ indicate an internal threshold and a normalization coefficient, respectively. Piéron's law results from a 
temporal sequence of events that differentiates those components near the threshold $S_{0}$ from those at suprathreshold conditions $\left(S>S_{0}\right)$. The coefficient $k$ follows a power law (Norwich et al., 1989; Norwich, 1993):

$$
k=t_{R T_{0}} S_{0}^{p}
$$

The asymptotic term $t_{R T_{0}}$ only contains the initial encoding time $t_{0}$ and $\beta S_{0}^{p}$, and it obeys a similar power law (Medina, 2012):

$$
t_{R T_{0}}=t_{0}\left(1+\beta S_{0}^{p}\right)
$$

Equation (2) corroborates that the coefficient $k$ has a direct link with a threshold mechanism in human vision (Plainis and Murray, 2000; Murray and Plainis, 2003; Medina and Diaz, 2005, 2006).

There is a chronological order that cannot be violated, namely, $t_{R T}>t_{R T_{0}}>t_{0}>0$. This is a direct consequence of $\Delta H$ and involves the principle of causality over time, which states that the effect cannot be before the cause. The formation of a threshold at $t_{R T_{0}}$ cannot precede the stimulus encoding at $t_{0}$, and those processes at suprathreshold conditions at $t_{R T}$ cannot precede those at $t_{R T_{0}}$ either (Medina et al., 2014). Further, Piéron's law is shape-invariant under rescaling (Chater and Brown, 1999) in a fractal-like process. In the rate domain (1/RT), Piéron's law has a direct link with the Naka-Rushton equation in neurophysiology (Naka and Rushton, 1966; Carandini and Heeger, 2012). Let, $R=$ $1 / t_{R T}$, and $R_{M}=1 / t_{R T_{0}}$, from Equations (1) and (2) (Medina, 2009):

$$
R=\frac{R_{M}}{1+\left(\frac{S_{0}}{S}\right)^{p}}
$$

Equations (2) and (3) show that threshold impairment in $S_{0}$ leads to longer RTs and consequently, it modifies Piéron's law in Equation (1). We exemplify the non-trivial effects of anomalous power law behavior $\beta S_{0}^{p}$ in Piéron's law in two different scenarios. $\beta, S_{0}$, and $p$ could vary based on several experimental factors. Similar examples follow in the same way. In the first example, we illustrate Piéron's law in amblyopia. Amblyopia (usually called "lazy eye") affects approximately $3 \%$ of human population and is a combination of visual deficits that impairs binocular vision from physiological alterations during early development (Ciuffreda et al., 1991; Howard, 2002). Figure 1A simulates the typical variation of the reciprocal of $S_{0}$ for spatial sine-wave gratings in normal and amblyopic vision. Threshold values $S_{0}$ are higher in the amblyopic eye at high spatial frequencies (Ciuffreda et al., 1991). This deficit is the principal responsible for higher $\beta S_{0}^{p}, k$, and $t_{R T_{0}}$ values in Equation (1) and consequently,

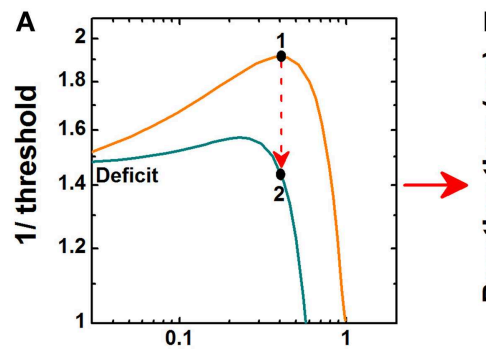

Spatial frequency (a.u.)
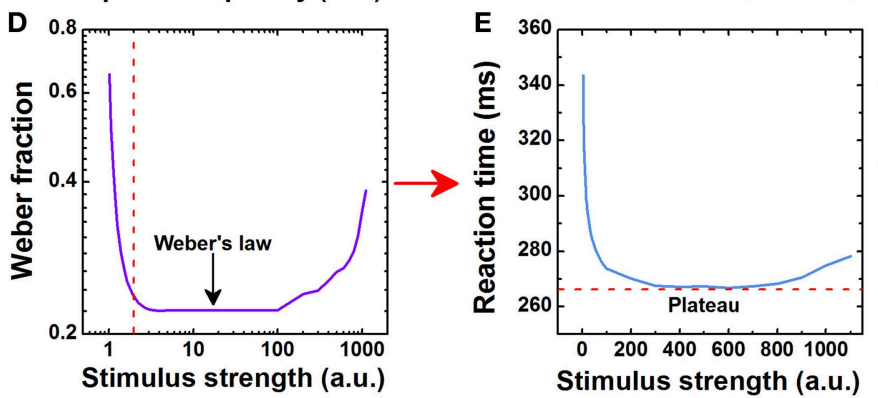
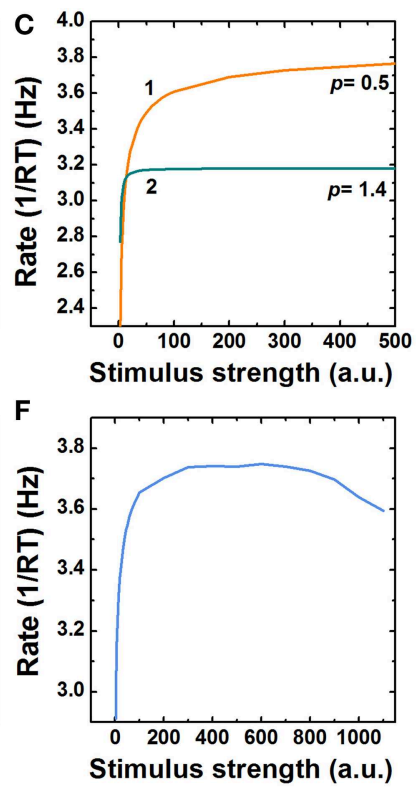

FIGURE 1 | Examples of Piéron's law. (A) Double logarithmic plot of the contrast sensitivity function (1/threshold) as a function of the spatial frequency for sine-wave gratings. Points labeled as "1" and "2" indicate two threshold values at the same spatial frequency that correspond to normal and amblyopic vision, respectively (Ciuffreda et al., 1991). In both cases the adapting conditions were the same and fixed. (B) Linear plot of Piéron's law as a function of the stimulus strength $S$. Vertical dash and dash-dot lines represent those threshold values labeled as "1" and "2" in (A), respectively. Solid lines labeled as "1" and "2" show the corresponding Piéron's law in normal and amblyopic vision, respectively (Pianta and Kalloniatis, 1998). Simulation parameters in normal vision: $t_{0}=44, \beta=294.39, S_{0}=0.52, p=0.5$. Amblyopic eye: $t_{0}=44$, $\beta=439.35, S_{0}=0.71, p=1.4$. (C) Linear plot of Piéron's law in the rate domain (1/RT). Solid lines labeled as "1" and "2" follow the same as in (B). (D) Double logarithmic plot of the Weber fraction $(\Delta S / S)$ as a function of intensity $S$. Vertical dash line separates the Rose-de Vries regime at low S-values from Weber's law. (E) Linear plot of Piéron's law as a function of the stimulus strength $S$. The adapting conditions now vary. The power law $\beta S_{0}^{p}$ is mapped onto a Weber fraction-type power law $(\Delta S / S)^{p}$ (Medina, 2011) and was varied from Weber's law to the terminal rise in (D). Simulation parameters: $t_{0}=150, \beta=1, p=0.33$. (F) Linear plot of Piéron's law in the rate domain. (a.u.)= arbitrary units. 
for longer RTs in amblyopic vision (Figure 1B) (Pianta and Kalloniatis, 1998). In the rate domain (Equation 4), amblyopic vision is limited because it gives saturated responses sooner (Figure 1C).

The second example illustrates the van der Mollen-Keuss effect in RTs. The van der Mollen-Keuss effect imposes a limitation to Piéron's law by producing a U-shaped function at very high $S$-values (van der Molen and Orlebeke, 1980; Jaśkowski and Włodarczyk, 2006; Marino and Munoz, 2009). $\beta S_{0}^{p}$ also depends on the sensory adaptation level (Plainis and Murray, 2000; Murray and Plainis, 2003; Medina, 2011). Figure 1D simulates the differential threshold relative to the background or Weber fraction $(\Delta S / S)$ as a function of the intensity $S$. The minimum value corresponds to Weber's law. There is a terminal rise at very high intensities. By interpreting the $\beta S_{0}^{p}$ as a Weber fraction, the terminal rise in Weber's law which is observed in many modalities gives rise to an abrupt increment in both $k$, and $t_{R T_{0}}$ in Equation (1) for high intensities. The van der Mollen-Keuss effect can therefore be explained theoretically

\section{References}

Baird, J. C. (1997). Sensation and Judgement. Mahwah, NJ: Lawrence Erlbaum Associates.

Carandini, M., and Heeger, D. J. (2012). Normalization as a canonical neural computation. Nat. Rev. Neurosci. 13, 51-62. doi: 10.1038/ nrn3136

Cattell, J. M. (1886). The influence of the intensity of the stimulus on the lenght of the reaction time. Brain 8, 512-515. doi: 10.1093/brain/8.4.512

Chater, N., and Brown, G. D. A. (1999). Scale-invariance as a unifying psychological principle. Cognition 69, B17-B24. doi: 10.1016/S0010-0277(98)00066-3

Ciuffreda, K. J., Levi, D. M., and Selenow, A. (1991). Amblyopia: Basic and Clinical Aspects. Boston, MA: Butterworth-Heinemann.

Donkin, C., and van Maanen, L. (2014). Piéron's law is not just an artifact of the response mechanism. J. Math. Psychol. 62-63, 22-32. doi: 10.1016/j.jmp.2014.09.006

Howard, I. P. (2002). Seeing in Depth. Toronto, ON: I. Porteous.

Hsu, Y. F. (2005). A generalization of Pieron's law to include background intensity and latency distribution. J. Math. Psychol. 49, 450-463. doi: 10.1016/j.jmp.2005.02.001

Jaśkowski, P., and Włodarczyk, D. (2006). Task modulation of the effects of brightness on reaction time and response force. Int. J. Psychophysiol. 61, 98-112. doi: 10.1016/j.ijpsycho.2005.07.010

Kello, C. T., Brown, G. D. A., Ferrer-i-Cancho, R., Holden, J. G., LinkenkaerHansen, K., Rhodes, T., et al. (2010). Scaling laws in cognitive sciences. Trends Cogn. Sci. 14, 223-232. doi: 10.1016/j.tics.2010.02.005

Link, S. (1992). The Wave Theory of Difference and Similarity. Hillsdale, NJ: Lawrence Erlbaum Association.

Luce, R. D. (1986). Response Times. New York, NY: Oxford University Press.

Marino, R., and Munoz, D. (2009). The effects of bottom-up target luminance and top-down spatial target predictability on saccadic reaction times. Exp. Brain Res. 197, 321-335. doi: 10.1007/s00221-009-1919-x

Medina, J. M. (2009). 1/f (alpha) noise in reaction times: a proposed model based on Pieron's law and information processing. Phys. Rev. E 79:011902. doi: 10.1103/PhysRevE.79.011902

Medina, J. M. (2011). Effects of multiplicative power law neural noise in visual information processing. Neural Comput. 23, 1015-1046. doi: 10.1162/NECO_a_00102

Medina, J. M. (2012). Multiplicative processes and power laws in human reaction times derived from hyperbolic functions. Phys. Lett. A 376, 1617-1623. doi: 10.1016/j.physleta.2012.03.025 as a consecuence of an entropy-based approach together with Weber's law (Figure 1E). In the rate domain (Equation 4), the reciprocal of RT shows an inverted U-shaped function (Figure 1F). This suggests a correlation with specific neural activity (Peirce, 2007). Outside the framework of Piéron's law, a more ellaborate approach to the Weber fraction and Weber's law has been developed using the same information-theoretic formalism (Norwich, 1993; Norwich and Wong, 1997).

Donkin and van Maanen fitted three different experimental data sets to test the validity of their assumptions (Donkin and van Maanen, 2014). Good fits to experimental data are neccesary but insufficient to support theoretical models with free parameters. Power laws in complex systems are better supported by models that constraint possible results and predict how experiments agree with such constraints (Roberts and Pashler, 2000; Kello et al., 2010; Stumpf and Porter, 2012). Hence, we have introduced a poweful approach for analyzing the relationship between an internal variable sensory threshold and Piéron's law by using information theory and power law scaling.

Medina, J. M., and Diaz, J. A. (2005). "Contrast coding in the parvo-cellula system using simple reaction times," in Proceedings of the 10th Congress of the International Colour Association, eds J. L. Nieves and J. Hernández-Andrés (Granada: Association Internationale de la Couleur- AIC), 179-182.

Medina, J. M., and Diaz, J. A. (2006). Postreceptoral chromatic-adaptation mechanisms in the red-green and blue-yellow systems using simple reaction times. J. Opt. Soc. Am. A 23, 993-1007. doi: 10.1364/JOSAA.23.000993

Medina, J. M., Díaz, J. A., and Norwich, K. (2014). A theory of power laws in human reaction times: insights from an information-processing approach. Front. Hum. Neurosci. 8:621. doi: 10.3389/fnhum.2014.00621

Murray, I. J., and Plainis, S. (2003). Contrast coding and magno/parvo segregation revealed in reaction time studies. Vision Res. 43, 2707-2719. doi: 10.1016/S0042-6989(03)00408-5

Naka, K. I., and Rushton, W. A. H. (1966). S-potentials from luminosity units in retina of fish (cyprinidae). J. Physiol. 185, 587-599. doi: 10.1113/jphysiol.1966.sp008001

Norwich, K. H. (1993). Information, Sensation, and Perception. San Diego, CA: Academic Press.

Norwich, K. H., Seburn, C. N. L., and Axelrad, E. (1989). An informational approach to reaction times. B. Math. Biol. 51, 347-358. doi: 10.1007/BF02460113

Norwich, K. H., and Wong, W. (1997). Unification of psychophysical phenomena: the complete form of Fechner's law. Atten. Percept. Psychophys. 59, 929-940. doi: 10.3758/BF03205509

Palmer, J., Huk, A. C., and Shadlen, M. N. (2005). The effect of stimulus strength on the speed and accuracy of a perceptual decision. J. Vision 5, 376-404. doi: $10.1167 / 5.5 .1$

Peirce, J. W. (2007). The potential importance of saturating and supersaturating contrast response functions in visual cortex. J. Vision 7,10 . doi: 1310.1167/7.6.13

Pianta, M. J., and Kalloniatis, M. (1998). Characteristics of anisometropic suppression: simple reaction time measurements. Atten. Percept. Psychophys. 60, 491-502. doi: 10.3758/bf03206869

Piéron, H. (1914). Recherches sur les lois de variation des temps de latence sensorielle en fonction des intensités excitatrices. Anneé Psychol. 20, 17-96. doi: 10.3406/psy.1913.4294

Pins, D., and Bonnet, C. (1997). Reaction times reveal the contribution of the different receptor components in luminance perception. Psychon. B. Rev. 4 359-366. doi: 10.3758/bf03210793

Plainis, S., and Murray, I. J. (2000). Neurophysiological interpretation of human visual reaction times: effect of contrast, spatial frequency and luminance. Neuropsychologia 38, 1555-1564. doi: 10.1016/S0028-3932(00)00100-7 
Roberts, S., and Pashler, H. (2000). How persuasive is a good fit? A comment on theory testing. Psychol. Rev. 107, 358-367. doi: 10.1037/0033-295X.107.2.358

Servant, M., Montagnini, A., and Burle, B. (2014). Conflict tasks and the diffusion framework: insight in model constraints based on psychological laws. Cogn. Psychol. 72, 162-195. doi: 10.1016/j.cogpsych.2014. 03.002

Stafford, T., and Gurney, K. N. (2004). The role of response mechanisms in determining reaction time performance: Pieron's law revisited. Psychon. B Rev. 11, 975-987. doi: 10.3758/BF03196729

Stafford, T., Ingram, L., and Gurney, K. N. (2011). Piéron's law holds during stroop conflict: insights into the architecture of decision making. Cogn. Sci. 35, 1553-1566. doi: 10.1111/j.1551-6709.2011.01195.x

Stumpf, M. P. H., and Porter, M. A. (2012). Critical truths about power laws. Science 335, 665-666. doi: 10.1126/science. 1216142

van der Molen, M. W., and Orlebeke, J. F. (1980). Phasic heart rate change and the $\mathrm{U}$-shaped relationship between choice reaction time and auditory signal intensity. Psychophysiology $17,471-481$. doi: 10.1111/j.14698986.1980.tb00186.x

Verdonck, S., and Tuerlinckx, F. (2014). The ising decision maker: a binary stochastic network for choice response time. Psychol. Rev. 121, 422-462. doi: $10.1037 / \mathrm{a} 0037012$

Conflict of Interest Statement: The authors declare that the research was conducted in the absence of any commercial or financial relationships that could be construed as a potential conflict of interest.

Copyright ( 2015 Medina and Díaz. This is an open-access article distributed under the terms of the Creative Commons Attribution License (CC BY). The use, distribution or reproduction in other forums is permitted, provided the original author(s) or licensor are credited and that the original publication in this journal is cited, in accordance with accepted academic practice. No use, distribution or reproduction is permitted which does not comply with these terms. 